\title{
Application of Aptamers Improves CRISPR-Based Live Imaging of Plant Telomeres
}

\author{
Solmaz Khosravi ${ }^{1}$, Patrick Schindele ${ }^{2}$, Evgeny Gladilin ${ }^{1}$, Frank Dunemann ${ }^{3}$, \\ Twan Rutten ${ }^{1}$, Holger Puchta ${ }^{2 *}$ and Andreas Houben ${ }^{1 *}$ \\ ${ }^{1}$ Department for Breeding Research, Leibniz Institute of Plant Genetics and Crop Plant Research (IPK), Seeland, Germany, \\ ${ }^{2}$ Botanical Institute, Karlsruhe Institute of Technology, Karlsruhe, Germany, ${ }^{3}$ Institute for Breeding Research on Horticultural \\ Crops, Julius Kühn-Institut (JKI), Quedlinburg, Germany
}

Development of live imaging techniques for providing information how chromatin is organized in living cells is pivotal to decipher the regulation of biological processes. Here, we demonstrate the improvement of a live imaging technique based on CRISPR/

OPEN ACCESS

Edited by:

Iva Mozgova,

Academy of Sciences of the Czech

Republic (ASCR), Czechia

Reviewed by:

Martina Dvorackova,

Central European Institute of

Technology (CEITEC), Czechia

Sachihiro Matsunaga,

The University of Tokyo, Japan

*Correspondence:

Holger Puchta

holger.puchta@kit.edu

Andreas Houben

houben@ipk-gatersleben.de

Specialty section: This article was submitted to

Plant Cell Biology,

a section of the journal

Frontiers in Plant Science

Received: 03 June 2020

Accepted: 30 July 2020

Published: 20 August 2020

Citation:

Khosravi S, Schindele P, Gladilin E, Dunemann F, Rutten T, Puchta $\mathrm{H}$ and

Houben A (2020) Application of

Aptamers Improves CRISPR-Based

Live Imaging of Plant Telomeres.

Front. Plant Sci. 11:1254.

doi: 10.3389/fp/s.2020.01254
Cas9. In this approach, the sgRNA scaffold is fused to RNA aptamers including MS2 and PP7. When the dead Cas9 (dCas9) is co-expressed with chimeric sgRNA, the fluorescent coat protein-tagged for MS2 and PP7 aptamers (tdMCP-FP and tdPCP-FP) are recruited to the targeted sequence. Compared to previous work with dCas9:GFP, we show that the quality of telomere labeling was improved in transiently transformed Nicotiana benthamiana using aptamer-based CRISPR-imaging constructs. Labeling is influenced by the copy number of aptamers and less by the promoter types. The same constructs were not applicable for labeling of repeats in stably transformed plants and roots. The constant interaction of the RNP complex with its target DNA might interfere with cellular processes.

Keywords: aptamer, CRISPR/dCas9, live imaging, $\boldsymbol{N}$. benthamiana, R-loops, telomere

\section{INTRODUCTION}

The 3D organization of the genome is involved in the regulation of various genomic functions including gene expression, transcription, DNA replication, and repair (Misteli, 2007). Different strategies have been developed to monitor the dynamics of defined genomic loci in living cells (Robinett et al., 1996; Lindhout et al., 2007; Chen et al., 2013; Saad et al., 2014; Fujimoto et al., 2016). Most recently, the clustered regularly interspaced short palindromic repeats (CRISPR)/CRISPR associated protein 9 (Cas9) based strategy has extensively been used mostly in non-plant species for live imaging. The first applications of CRISPR/Cas for live-cell imaging in plant (Dreissig et al., 2017; Fujimoto and Matsunaga, 2017) and non-plant cells (Chen et al., 2013) was based on fluorescent proteins directly fused to deactivated Cas9 (dCas9). Different dCas9 orthologues from Streptococcus pyogenes and Staphylococcus aureus successfully label telomeres in transiently transformed Nicotiana benthamiana leaves (Dreissig et al., 2017; Fujimoto and Matsunaga, 2017). Accordingly, it was shown that the locations of telomeres are in the periphery of the nucleus and dynamic positional changes of telomeres up to $\pm 2 \mu \mathrm{m}$ were reported (Dreissig et al., 2017). 
Indirect labeling of dCas9 with the SunTag method resulted in 19 fold brighter signals in mammalian cell cultures in comparison to GFP-fused dCas9 (Tanenbaum et al., 2014). However, this method like directly labeled dCas9 does not have the possibility of multi-targeting of genomic regions. For this purpose, different variants of dCas9 which have specific cognate gRNA were combined to label different genomic regions (Esvelt et al., 2013; Ma et al., 2015; Dreissig et al., 2017). To improve the efficiency of imaging and also the capacity of dCas9 for multi-targeting of different regions at the same time, other methods for indirect labeling of dCas9 were adapted including BIFC (Tanenbaum et al., 2014; Hong et al., 2018), Aio-Casilio (Zhang and Song, 2017) and RNA-aptamer-based methods (Fu et al., 2016; Ma et al., 2016; Shao et al., 2016; Wang et al., 2016; Qin et al., 2017). CRISPR-based live-cell imaging methods are reviewed in (Wu et al., 2019; Khosravi et al., 2020).

Among the improved indirect labeling methods, aptamerbased methods are used in mammalian cell cultures to target telomeric and other genomic regions. Aptamers are short RNA oligos which can be detected by specific RNA binding proteins (Urbanek et al., 2014). Aptamer-based imaging methods are based on three components including dCas9, sgRNA in which the aptamer sequence is integrated and the aptamer binding protein which is fused to the fluorescent protein (Fu et al., 2016; Ma et al., 2016; Shao et al., 2016). In plants, aptamers have been used for CRISPR/Cas9 targeted gene regulation with effector proteins like transcription activation domains, acetyltransferase or methyltransferase which were fused to the aptamer binding protein (Lee et al., 2019; Selma et al., 2019). The copy number of aptamers determines the number of effector proteins enriched in the targeted region. However, no application of CRISPR live-cell imaging based on aptamers is reported in plants yet.

In this research, we developed a CRISPR life imaging method based on the application of MS2 and PP7 aptamers for targeting telomeres in transiently transformed $N$. benthamiana. We investigate whether the copy number of aptamers, sgRNA scaffold changes and promoter type affect labeling efficiency of target sequences. However, the same method was not successful for constant labeling of chromosome regions in stably transformed plants ( $N$. benthamiana and A. thaliana) and roots (Daucus carota), suggesting that a continuous interaction of the RNP complex with target sequences might interfere with the progression of the cell cycle and plant development.

\section{MATERIALS AND METHODS}

\section{Plasmid Construction}

\section{Expression of dCas9 Driven by Different Promoters}

To establish a three-component aptamer-based labeling method, dCas9 under the control of a ubiquitin parsley promoter was indirectly labeled with aptamer binding proteins (MS2 or PP7) fused to fluorescent proteins. The $35 \mathrm{~S}$ promoter was amplified with EcoRI-35S-f1 and r1 primers flanking with an EcoRI recognition site from pCCNCEN using Q5 DNA polymerase under following conditions: $98^{\circ} \mathrm{C}$ for $2 \mathrm{~min}, 30 \mathrm{x}\left(98^{\circ} \mathrm{C}\right.$ for $10 \mathrm{~s}$, $58^{\circ} \mathrm{C}$ for $30 \mathrm{~s}, 72^{\circ} \mathrm{C}$ for $30 \mathrm{~s}$ ) and $72^{\circ} \mathrm{C}$ for $2 \mathrm{~min}$. (Supplementary

Table 1). Then it was digested with EcoRI and cloned to linearized pDe-Sp-dCas9 GentR with EcoRI, which had another EcoRI site in the backbone and was removed in advance by site-directed mutation. The same method was used for substitution of the ubiquitin parsley promoter with a RPS5A promoter. The isolation of RPS5A was done by PRS5A-FWD and REV primers from the pGPTV-BAR using Q5 DNA polymerase under following conditions: $98^{\circ} \mathrm{C}$ for $2 \mathrm{~min}, 30 \mathrm{x}\left(98^{\circ} \mathrm{C}\right.$ for $10 \mathrm{~s}, 59^{\circ} \mathrm{C}$ for $30 \mathrm{~s}$, $72^{\circ} \mathrm{C}$ for $40 \mathrm{~s}$ ) and $72^{\circ} \mathrm{C}$ for $2 \mathrm{~min}$ (Supplementary Table 1). The XVE inducible promoter was generated with primers (Cas9-XVE$\mathrm{F}$ and XVE-Lexa-A-R; XVE-Lexa-A-F and LexA-Cas9-R (Supplementary Table 1) containing homologous flanks for further Gibson Assembly into the pDe-Sp-dCas9 GentR. Following PCR conditions was used for amplification with XVELexa-A-F and LexA-Cas9-R primers: $98^{\circ} \mathrm{C}$ for $2 \mathrm{~min}, 30 \mathrm{x}\left(98^{\circ} \mathrm{C}\right.$ for $10 \mathrm{~s}, 65^{\circ} \mathrm{C}$ for $30 \mathrm{~s}, 72^{\circ} \mathrm{C}$ for $20 \mathrm{~s}$ ) and $72^{\circ} \mathrm{C}$ for $2 \mathrm{~min}$. For amplification with Cas9-XVE-F and XVE-Lexa-A-R primers, the same conditions were used except the extension time which was increased to $2 \mathrm{~min}$. The pER8-v3 plasmid was used for generation of the XVE inducible promoter (Zuo et al., 2000) (Supplementary Table 1). According to (Dreissig et al., 2017), a pChimera expression gRNA vector in combination with a dCas9-eGFP expression vector was used as a control vector to target telomeres.

\section{Insertion of Aptamer Sequences Into the sgRNA Scaffold}

For aptamer-mediated imaging, sgRNA expression vectors were created either harbouring one MS2 aptamer sequence each in the tetraloop and stem-loop 2 of the S. pyogenes sgRNA backbone (Konermann et al., 2015) or three PP7 aptamer sequences only in the tetraloop of the $S$. pyogenes sgRNA backbone additionally comprising an A-U pair flip and stem extension (Shechner et al., 2015). In case of MS2, the vector pDS2.0-MS2 was synthesized comprising the respective sgRNA under control of the AtU6-26 promoter together with the codon-optimized MS2 binding protein cds joined to a 3' SV40 NLS by a 3x GGGGS linker under control of the ZmUbi-1 promoter. In case of PP7, the respective sgRNA and codon-optimized PP7 binding protein cds also harboring a 3' SV40 NLS were synthesized and subcloned via restriction digestion and ligation into pDS2.0-MS2 creating pDS2.0-PP7. BsmBI restriction sites downstream of the aptamer binding protein cds were used for in-frame cloning of a 3-fold fusion of either eGFP or mRuby2. For this purpose, the respective cds were amplified from pSIM24-eGFP and pcDNA3-mRuby2 (www.addgene.com) with primers (MS2(NLS)-GFP\#1-f, GFP\#1linker1-r, linker1-GFP\#2-f, GFP\#2-linker2-r, linker2-GFP\#3-f, GFP\#3-nos_ter-r or MS2(NLS)-mRuby\#1-f, mRuby\#1-linker1-r, linker1-mRuby\#2-f, mRuby\#2-linker2-r, linker2-mRuby\#3-f, mRuby\#3-nos_ter-r) adding homologous flanks for subsequent Gibson Assembly into the linearized pDS2.0-MS2 or pDS2.0-PP7 similar as previously described (Dreissig et al., 2017) creating pDS2.0-MS2/PP7-3xeGFP/3xmRuby2 (Supplementary Table 1). 


\section{Changing the sgRNA Scaffold}

An MS2 aptamer-harboring sgRNA additionally comprising an A-U flip and stem extension (Chen et al., 2013) was synthesized and subcloned into pDS2.0-MS2-eGFP/mRuby2. For this purpose, pDS2.0-MS2-eGFP/mRuby2 was amplified with primers (pDS2.0- $\Delta$ sgRNA-r, pDS2.0- $\Delta$ sgRNA-f) deleting the sgRNA and the synthesized sgRNA was amplified with primers (sgRNA2.0-MS2-flip/ext-f, sgRNA2.0-MS2-flip/ext-r) adding overhangs for subsequent Gibson Assembly into the linearized backbone (Supplementary Table 1).

\section{Altering the Copy Number of Aptamers}

To change the copy number of aptamers, pDS.2.0-MS2+3xeGFP gRNA expression vector was used. To delete one of MS2 copy numbers, pDS.2.0-MS2+3xeGFP was double digested with Agel and $M s c$ I restriction enzymes and then was ligated to annealed primers Apta2-FWD and Apta2-Rev flanked by Agel overhang (Supplementary Table 1). Annealing of primers was done by mixing $2 \mu \mathrm{l}$ of each primer $(100 \mathrm{pM})$ in the total volume of $50 \mu \mathrm{l}$ double distilled water and incubation at $95^{\circ} \mathrm{C}$. Colony PCR was performed by SS42 and Apta2-Rev2 primers under following conditions: $95^{\circ} \mathrm{C}$ for $5 \mathrm{~min}, 30 \mathrm{x}\left(95^{\circ} \mathrm{C}\right.$ for $30 \mathrm{~s}, 58^{\circ} \mathrm{C}$ for $30 \mathrm{~s}, 72^{\circ} \mathrm{C}$ for $30 \mathrm{~s}), 72^{\circ} \mathrm{C} 5 \mathrm{~min}$. Positive clones were confirmed by sequencing with the SS42 primer (Supplementary Table 1). To increase the copy number of aptamer sequences, a pDS2.0-MS2eGFP/mRuby2 sgRNA expression vector was used. First, according to Qin et al., 2017 a sgRNA scaffold harbouring 16 MS2 aptamers was synthesized and subcloned into pDS2.0-MS2$\mathrm{eGFP} / \mathrm{mRuby} 2$. For this purpose, pDS2.0-MS2-eGFP/mRuby2 was digested with BsmBI and AgeI for sgRNA deletion and the synthesized sgRNA was digested with BsaI and AgeI for subsequent ligation into the linearized pDS2.0-MS2-eGFP/ mRuby2 creating pDS2.0-16xMS2-eGFP/mRuby2.

\section{Designing Protospacers for Targeting Different Genomic Regions}

The protospacer design was performed with the help of DeskGen (https://www.deskgen.com/). Each protospacer sequence was selected based on the PAM sequence of SpCas9 and synthesized as primer oligos with appropriate overhangs at 5 ' ends for cloning into the pDS2.0-MS2:3xeGFP/mRuby (Supplementary Table 1). Then, the pDS2.0-MS2:3xeGFP/ mRuby was subcloned to dCas9 expression vector by Gateway cloning. The dCas9 expression vector carries a gentamycin resistant marker for selection of stably transformed plants. The telomere protospacer was designed based on Arabidopsis-type telomere repeat sequence 5'-(TTTAGGG)(n)-3'. Arabidopsistype centromere-specific protospacers were designed based on centromeric satellite consensus sequences (Supplementary Table 1).

\section{Plant Material and Transformation}

All imaging constructs were separately transformed to Agrobacterium tumefaciens GV3101. For carrot transformation, A. rhizogenes 15843 was used. Agrobacteria were cultured overnight at $28^{\circ} \mathrm{C}$ in LB medium containing spectinomycin (100 $\left.\mathrm{mg} / \mathrm{l}^{-1}\right)$ and rifampicin $\left(50 \mathrm{mg} / \mathrm{l}^{-1}\right)$ for transient transformation of
N. benthamiana according to (Phan and Conrad, 2016). Additionally, a N. benthamiana line expressing CFP-histone H2B was used (Martin et al., 2009). For the telomeric repeat binding protein 1 fused to GFP (TRB1-GFP), Agrobacteria were cultured in LB medium containing kanamycin $\left(100 \mathrm{mg} / \mathrm{l}^{-1}\right)$ and rifampicin $\left(50 \mathrm{mg} / \mathrm{l}^{-1}\right)$ (Schrumpfová et al., 2014). For cotransformation experiments, bacterial cultures with the same $\mathrm{OD}_{600}(0.5)$ were mixed in a 1:1 ratio. Stable transformation of $N$. benthamiana, D. carota (cultivars Blanche, Yellowstone and Rotin) and $A$. thaliana (var. Columbia) with dCas9:2xMS2:GFP constructs were performed via leaf samples, A. rhizogenes-based hairy root transformation and floral dip method according to (Clemente, 2006), (Dunemann et al., 2019) and (Martin et al., 2009), respectively. PCR $\left(95^{\circ} \mathrm{C}\right.$ for $5 \mathrm{~min}, 30 \mathrm{x}\left(95^{\circ} \mathrm{C}\right.$ for $30 \mathrm{~s}, 58^{\circ} \mathrm{C}$ for $30 \mathrm{~s}, 72^{\circ} \mathrm{C}$ for $\left.30 \mathrm{~s}\right), 72^{\circ} \mathrm{C}$ for $\left.5 \mathrm{~min}\right)$ and real-time PCR $\left(95^{\circ} \mathrm{C}\right.$ for $10 \mathrm{~min}, 40 \mathrm{x}\left(95^{\circ} \mathrm{C}\right.$ for $10 \mathrm{~s}, 60^{\circ} \mathrm{C}$ for $\left.1 \mathrm{~min}\right)$ and melt curve stage of $95^{\circ} \mathrm{C}$ for $30 \mathrm{~s}, 60^{\circ} \mathrm{C}$ for $15 \mathrm{~s}$ ) were performed for putative transgenic plants using primers specific for dCas9 and GFP to confirm the presence and expression of T-DNA fragments (Supplementary Table 1).

\section{Immunostaining and Fluorescence In Situ Hybridization (FISH)}

Sampling for immunostaining was performed three days after infiltration of $N$. benthamiana. Briefly, a piece of leaf tissue with the size of $\sim 1 \mathrm{~cm}^{2}$ was excised and chopped in $0.5 \mathrm{ml}$ chromosome isolation buffer (Doležel et al., 2007) and then filtrated through a $35 \mu \mathrm{m}$ nylon mesh with subsequent centrifugation onto microscopic slides with a CytoSpin3 (Shandon) at $400 \mathrm{rpm}$ for $5 \mathrm{~min}$. To confirm the specificity of signals CRISPR imaging and FISH were combined. The intensity of CRISPR signals was increased by in direct immunostaining using a 1:2,500 diluted Dylight 488-labeled GFP mouse monoclonal antibody (cat. 200-341-215, Rockland) according to (Ishii et al., 2015). Detection of Arabidopsis-type telomeres via FISH was performed with a $5^{\prime}$ Cy5-labeled probe $\left(5^{\prime}\right.$ GGGTTTAGGGTTTAGGGTTT). Immuno-FISH was performed as described by (Ishii et al., 2015). Immunostaining against dCas9, was performed with a DyLight 550-labeled SpCas9 mouse monoclonal antibody (cat. NBP2-52398R, Novus Biological).

\section{Proteasome Inhibitor Test}

The plants were kept on MS medium containing 50,100, or $150 \mu \mathrm{M}$ MG-132 (Serva) under dark condition at room temperature for $16 \mathrm{~h}$.

\section{Microscopy}

Micrographs were captured using an epifluorescence microscope (Olympus BX61) equipped with a cooled charge coupled device (CCD) camera (Orca ER; Hamamatsu). Images were collected from at least 10 nuclei per experiment and then analyzed with ImageJ. For live-cell imaging, a confocal laser scanning microscope (LSM780, Carl Zeiss) was used. To detect fluorescence signals in vivo, a piece of infiltrated leaf was cut and with the use of $40 \mathrm{x}$ NA 1.2 water objective nuclei with clear signals were tracked for $20 \mathrm{~min}$. 488-nm laser line was used for 
excision of GFP and emission was detected over a range of 490$540 \mathrm{~nm}$.

\section{Statistics}

For statistical analysis the program package SigmaStat 4.0 was used (Systat Software, Inc.; https://systatsoftware.com/). Oneway ANOVA followed by pairwise comparison was used for more than two samples and two-tailed Student's t-test was used for comparison of two samples.

\section{Analysis of Telomere Signals}

To measure the labeling efficiency of telomeres, 20 nuclei were imaged for each construct by epifluorescent microscope. The number of telomere signals per nucleus was determined and the mean value was calculated. To evaluate the signal/ background noise, the maximum signal intensity was divided by minimum signal intensity rising from the background using the ImageJ software. The mean value was calculated from three measurements in each nucleus.

To study the movement of telomeres, telomere tracking was performed for 5 nuclei and was based on time-laps $\mathrm{z}$ stacks from IMARIS 8.0 (Bitplane). The adjustments to calculate the coordinates $(x, y, z)$ of each telomere and also measuring the inter-telomere distances was based on Dreissig et al. (2017). To assess true displacements of telomeres over time, global movements of nuclei have to be computationally eliminated. For this purpose, $3 \mathrm{D}$ point clouds of telomere mass centres for all subsequent time steps $(t>0)$ were rigidly registered to the reference system of coordinates defined by the first time step $(t=0)$ using absolute orientation quaternions (Horn, 1987). To quantify the intranuclear telomere motion, the mean square distance $(M S D)$ of telomeres relatively to their initial position $(\mathrm{t}=0)$ was calculated as

$$
\operatorname{MSD}(t)=\frac{1}{N} \sum_{i=1}^{N}\left(R_{i}(t)-R_{i}(0)\right)^{2}
$$

where $R_{i}(t)$ is the radius vector of the $i$-th registered telomere in the reference system of coordinates at the time point $t>0$.

\section{RESULTS}

\section{Optimizing Live Imaging of Telomeres With Aptamer-Based CRISPR/dCas9 Imaging Vectors}

The application of fluorescent proteins directly fused to dCas 9 resulted in the labeling of $\sim 27$ telomeres of 72 expected signals in 2C nuclei of N. benthamiana (Dreissig et al., 2017). To improve the labeling efficiency, we established RNA aptamer-based CRISPR/dCas9 imaging constructs for plants. The three-component constructs (called dCas9:2xMS2:GFP and dCas9:3xPP7:GFP) encode dCas9 of $S$. pyogenes, an Arabidopsis telomere-specific sgRNA with integrated aptamer sequences (2x MS2 or 3x PP7) and aptamer coat proteins fused to three copies of fluorescent proteins (tdMCP : GFP or tdPCP : GFP binding to MS2 or PP7 aptamers, respectively) (Figures 1A, B). In addition, a dCas9:2xMS2 construct with a $3 \times$ mRubytagged coat protein (called dCas9:2xMS2:mRuby) was prepared (Supplementary Figure S1).

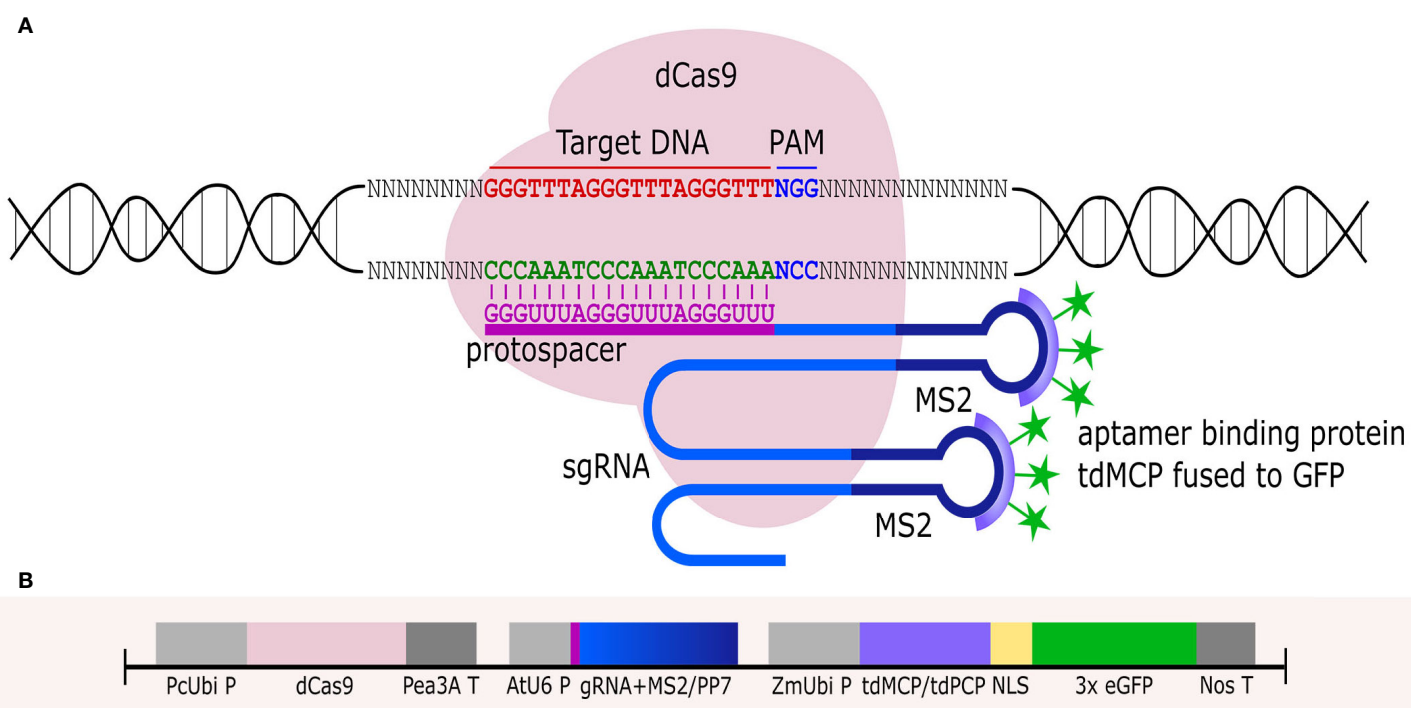

FIGURE 1 | RNA aptamer-based CRISPR/dCas9 imaging of telomere repeats. (A) Schemata depicting the components of the aptamer-based CRISPR labeling method: (1) dCas9 from S. pyogenes, (2) MS2 or PP7 aptamers (here only MS2 is shown) which are integrated into the sgRNA scaffold. (3) RNA binding protein (tdMCP or tdPCP) fused to fluorescent protein (3x eGFP) which recognizes aptamers. Protospacer designed to target Arabidopsis-type telomere DNA sequence. (B) Structure of the aptamer-based CRISPR imaging construct. dCas9 is driven by a ubiquitin promoter from parsley (PcUbi P), chimeric gRNA including aptamers (MS2/PP7) are driven by the AtU6 promoter (AtU6 P), aptamer binding proteins fused to a fluorescent protein (tdMCP/tdPCP) with the help of nuclear localization signal (NLS) are driven by a ubiquitin promoter from maize (ZmUbi P). Pea3A T and Nos T were used as terminators. 
To compare the labeling efficiency of the newly designed constructs, $N$. benthamiana leaves were separately infiltered with both types of Arabidopsis-type telomere-specific dCas9-aptamer constructs (dCas9:2xMS2:GFP and dCas9:3xPP7:GFP) and the previously employed dCas9:GFP reporter (Dreissig et al., 2017). Both types of aptamer-based constructs successfully labeled telomeres in interphase nuclei (Figures 2B, C). In average, 48 and 37 signals were recognized by dCas9-2xMS2:GFP and dCas9-3xPP7:GFP, respectively (Figure 2D). In contrast, the application of dCas9:GFP resulted in $\sim 28$ CRISPR-based signals which is consistent with earlier research (Dreissig et al., 2017) (Figures 2C, D). The lower number of detected signals than the expected could be due to clustering of some telomeres or not all telomeres were detectable by the applied imaging constructs. Notably, the accumulation of GFP signals in the nucleolus, which was always observed by application of dCas9:GFP was not found in nuclei labeled with both types of dCas9-aptamer constructs (Figures 2A-C).

As a negative control, the transformation of $N$. benthamiana with partial constructs carrying dCas9:GFP without target-specific gRNA or pMS2:mRuby targeting telomeres without the dCas9 component was performed. For both, a nonspecific labeling of nuclei was found (Figures 3A, B). After co-transformation with both partial constructs, overlapping telomere-like signals of green and red fluorescence were found due to the presence of all components required for CRISPR imaging of telomeres (Figure 3C).

To confirm the target specificity of the observed telomerelike signals, FISH with a labeled telomere-specific probe was performed after CRISPR imaging. All dCas9:2xMS2:GFP signals co-localized with FISH signals, demonstrating the target specificity of the aptamer-based imaging approach (Figure 4A). However, the labeling efficiency of CRISPR was less than FISH as only $78 \%$ and $75 \%$ of FISH signals colocalized with
dCas9:2xMS2:GFP and dCas9:3xPP7:GFP signals, respectively (Figure 4B). Co-expression of dCas9:2xMS2:mRuby with TRB1 and telomeric dCas9:2xMS2:GFP with CFP labeled histone H2B (Martin et al., 2009) showed that the aptamer-based CRISPR imaging method can also be successfully combined with fluorescence-labeled proteins to study DNA-protein interactions (Supplementary Movies S1 and S2).

To test whether the copy number of aptamers affects the labeling efficiency, we compared dCas9:MS2:GFP carrying 1, 2, or 16 copies of the MS2 aptamer. By reducing the aptamer copy number to 1, the number of observed signals reduced (Figure 5A). 16 copies of MS2 did not result in enhanced telomere signals, instead strong background signals were produced (Figure 5C).

Because four sequential $U$ nucleotides in the sgRNA stemloop could be recognized as a transcription termination signal for the A. thaliana derived U6 pol-III promoter, a U to A substitution was performed and also the structure of sgRNA was changed by the insertion of an extension to improve the stability of sgRNA and its assembly with dCas9 according to (Supplementary Figure S2). The U/A flip along with increasing the length of the sgRNA stem size did not result in a significant increase of telomere signal intensity and did not improve the signal/background noise ratio of telomere signals in $N$. benthamiana (Figures 6A-C).

\section{Comparing the Effect of Different Promoters to Express dCas9}

Beside the ubiquitin promoter from parsley to drive the expression of dCas9 in $N$. benthamiana, we tested the cauliflower mosaic virus (CaMV) 35S (Tepfer et al., 2004), RPS5A (Weijers et al., 2001) and the $\beta$-estradiol inducible promoter XVE (Zuo et al., 2000). Changing the promoter in dCas9:2xMS2:GFP construct did not increase the number of
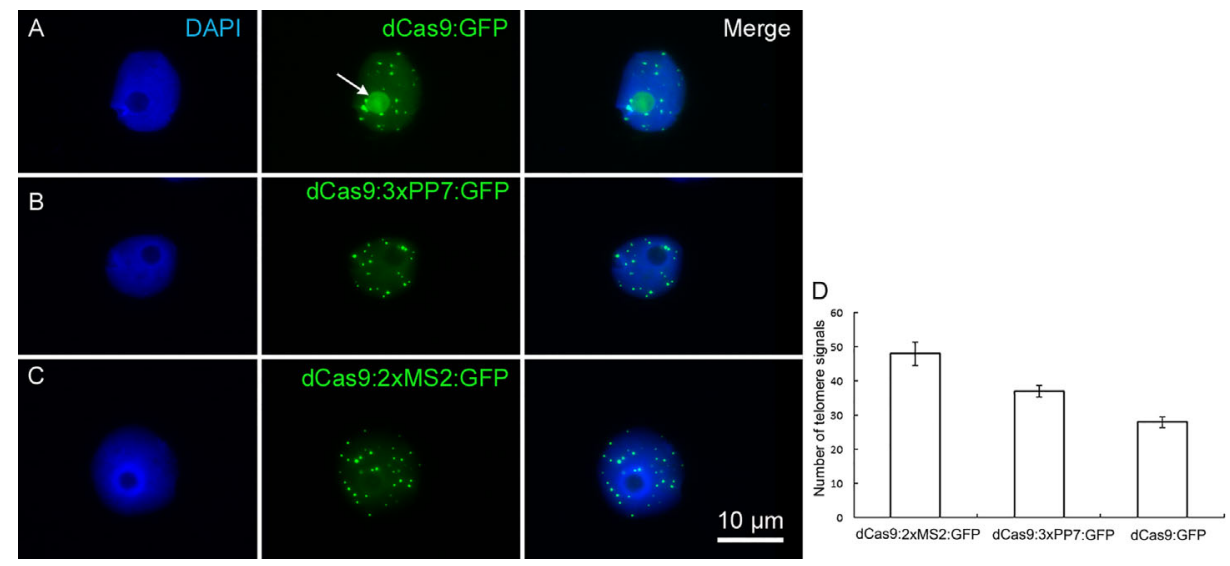

FIGURE 2 | Live imaging of telomeres in N. benthamiana leaf cells during interphase by CRISPR/dCas9. The distribution of telomeres recognized by (A) dCas9: GFP, (B) dCas9:3xPP7:GFP, and (C) dCas9:2xMS2:GFP. Note, aptamer-based imaging constructs (dCas9:3xPP7:GFP and dCas9:2xMS2:GFP) did not label nucleoli, while the application of dCas9:GFP does (nucleolus shown with white arrow). Nuclei are counterstained with DAPI (1.5 $\mu \mathrm{g} / \mathrm{ml}$ ) in VECTASHIELD.

(D) Diagram showing the efficiency of indirectly and directly labeled dCas 9 for targeting telomeric regions. The number of telomere signals was determined based on 20 nuclei per construct. dCas9 indirectly labeled either with MS2 or PP7 aptamers shows more telomeres $(p<0.05)$. 

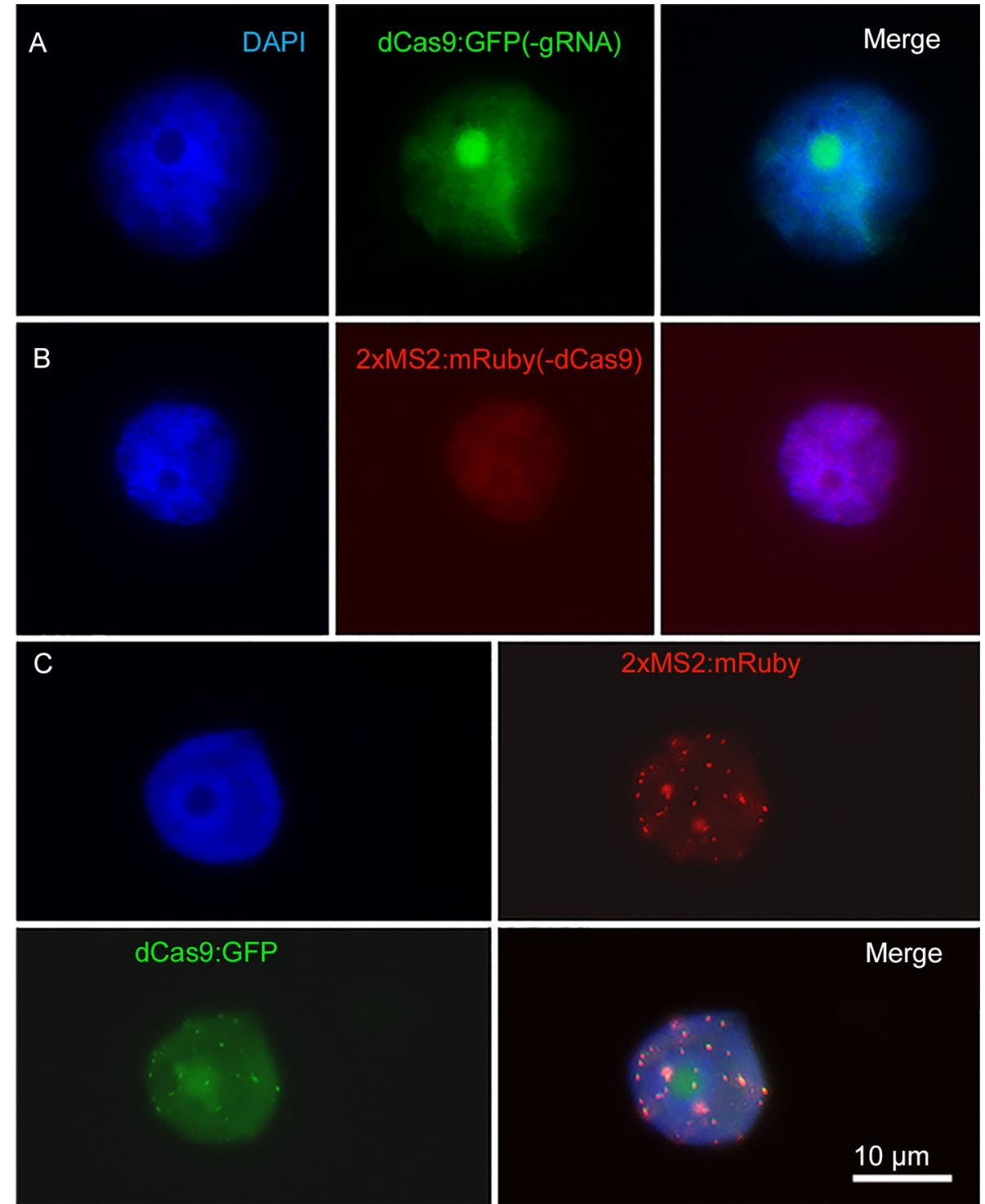

FIGURE 3 | Negative control with partial constructs carrying (A) dCas9:GFP without gRNA or (B) 2xMS2:3xmRuby targeting telomeres without dCas9. (C) Cotransformation of $N$. benthamiana leaves with both partial dCas9:GFP and 2xMS2:3xmRuby constructs resulted in labeling of telomeres, while no telomere-like signals were found after transformation with either partial construct (A, B). Nuclei are counterstained with DAPI.

observed telomere signals in comparison to the ubiquitin promoter (Figure 7A). The $35 \mathrm{~S}$ promoter led to a better signal/background noise ratio (Figure $7 \mathbf{B}$ ). After induction of the $\beta$-estradiol inducible XVE promoter, the same number of telomere signals was observed which was recognized by the construct driven by the ubiquitin promoter (Figure 7A). Regardless of promoter type, dCas 9 could label the telomeric regions in $N$. benthamiana (Figures $7 \mathrm{C}-\mathbf{E}$ ). The specificity of signals was approved by subsequent FISH with a telomerespecific probe (Supplementary Figure S3A). Without induction, no telomere-specific signal was observed (Supplementary Figure S3B).
Comparison of dCas9 transcription driven by the XVE or ubiquitin promoter revealed that even weak dCas 9 expression by XVE is sufficient to produce telomere-specific CRISPR-based signals (Supplementary Figure S4). Regardless of the promoter type, telomeres showed similar dynamic and random movements (Figure 8). To quantify these movements the mean square displacement (MSD) of telomeres was measured over a period of time. Calculating the changes of intratelomeric distance showed the minimum $\pm 1 \mu \mathrm{m}$ to maximum $\pm 4 \mu \mathrm{m}$ of changes for each type of promoter (Figure 9). In summary, application of RNAaptamers for CRISPR-based live-cell imaging increases the efficiency of telomere labeling in plant cells. 

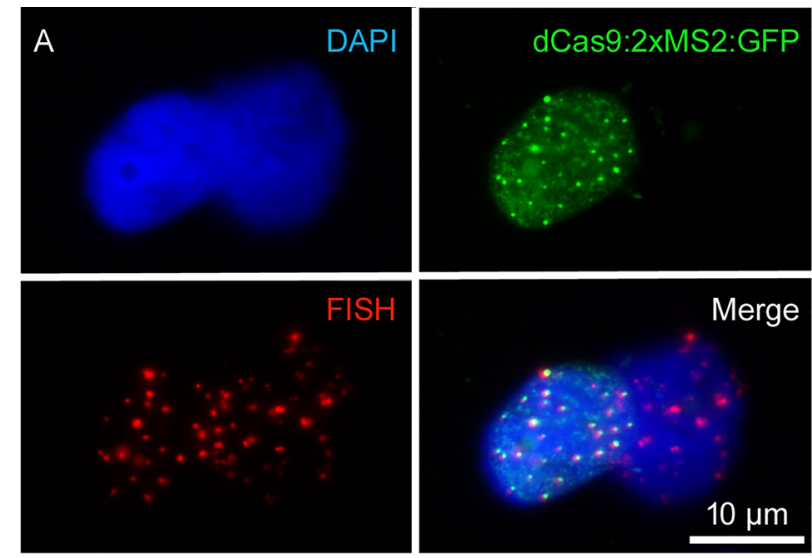

B

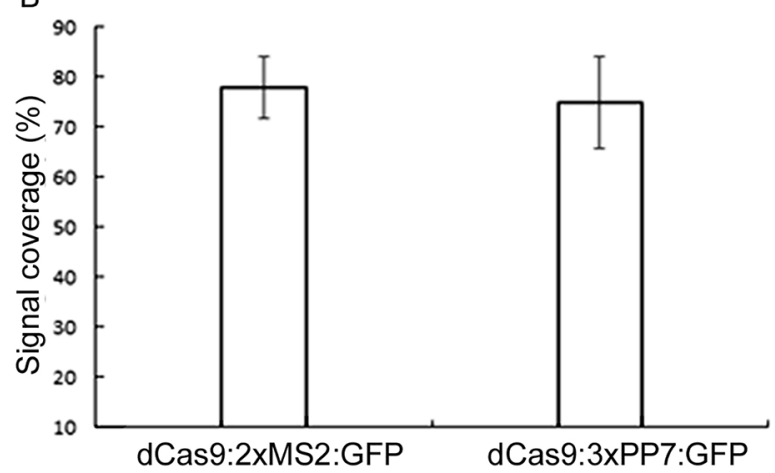

FIGURE 4 | Confirming the target specificity of aptamer-based CRISPR imaging (A) Immunofluorescence staining against dCas9:2xMS2:GFP combined with telomere-specific FISH. Nuclei are counterstained with DAPI (in blue).

(B) Comparing the efficiency of both types of aptamer-based CRISPR imaging with FISH. Telomeric signals based on 20 isolated nuclei per each construct after ImmunoFISH. dCas9:2xMs2:GFP and dCas9:3xPP7:GFP recognized 78\% and $75 \%$ of telomere signals identified by FISH, respectively $(p<0.05)$.

\section{Application of CRISPR-Imaging Is Limited in Stably Transformed Plants}

Stable transformation of $N$. benthamiana, A. thaliana plants and D. carota roots with the telomere-specific dCas9:2xMS2:GFP construct did not result in transgenic plants exhibiting GFPlabeled telomeres in living leaf or root cells, although the presence and expression of dCas9 and GFP genes were confirmed by PCR and real-time RT-PCR (data not shown, Supplement Table 2). Only transformation of A. thaliana with dCas9:2xMS2:GFP targeting centromeric regions resulted in few plants that showed some dot-like signals, however, the number and pattern of signals were atypical for interphase centromeres (Supplementary Figure S5). In total, 141 selection marker resistant $A$. thaliana plants were screened for three different centromere imaging constructs by microscopy. Among them, 27 plants showed uniform labeling of nuclei and 9 plants showed dot-like signals. The dot-like signals were unstable and could not be detected in seedlings older than three weeks or subsequent generations (T3). Phenotype and seed setting of plants exhibiting dot-like signals were wild-type like. Among the three different protospacers used, only protospacer 1 and 2 produced signals. The same protospacer 1 was successfully used to label centromeres in fixed nuclei of $A$. thaliana with the help of CRISPR-FISH (Ishii et al., 2019).

Plants that were transformed with dCas9:2xMS2:GFP under the control of an inducible promoter with a centromere- or telomere-specific protospacer revealed no target sequencespecific signals after induction with $\beta$-estradiol (Supplementary Table S2).

To test whether the disappearance of dot-like signals is caused by degradation of the dCas 9 protein, transgenic plants were treated with different concentrations of the proteasome inhibitor MG132. However, no dot-like signals were recovered. Additionally, the presence of dCas 9 protein was confirmed by dCas 9 immunostaining (Supplementary Figure S6).
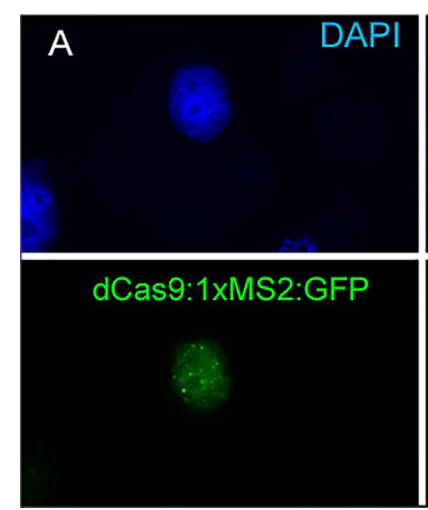

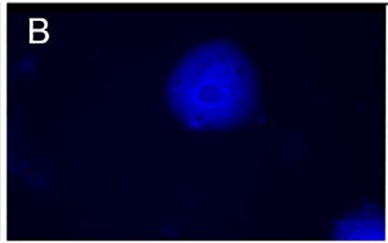

dCas9:2xMS2:GFP

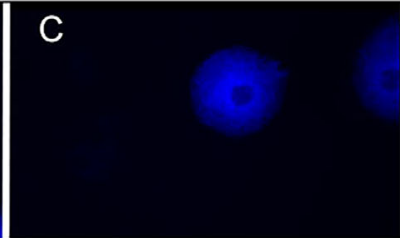

dCas9:16xMS2:GFP

$10 \mu \mathrm{m}$

FIGURE 5 | Effect of MS2 aptamer copy number of aptamer-based CRISPR imaging constructs on signal intensity. (A) dCas9:1xMS2, (B) dCas9:2xMS2, and (C) dCas9:16xMS2. The construct with two copies of MS2 revealed the best labeling of telomeres. Nuclei are counterstained with DAPI. 
A

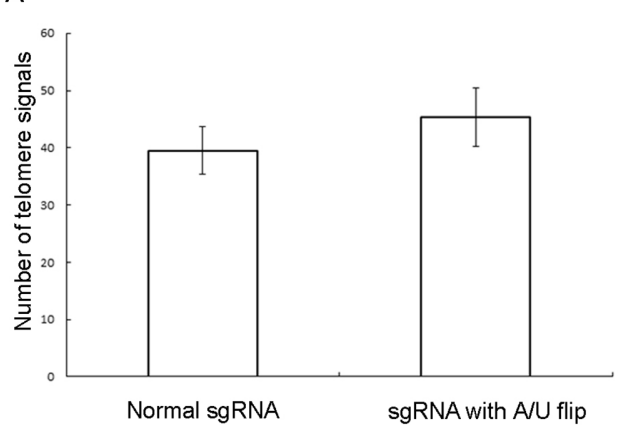

B

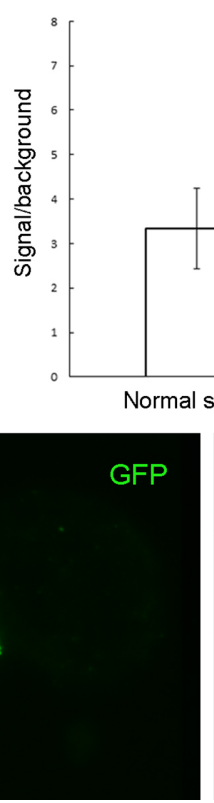

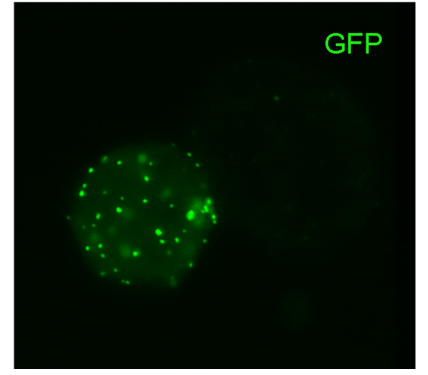
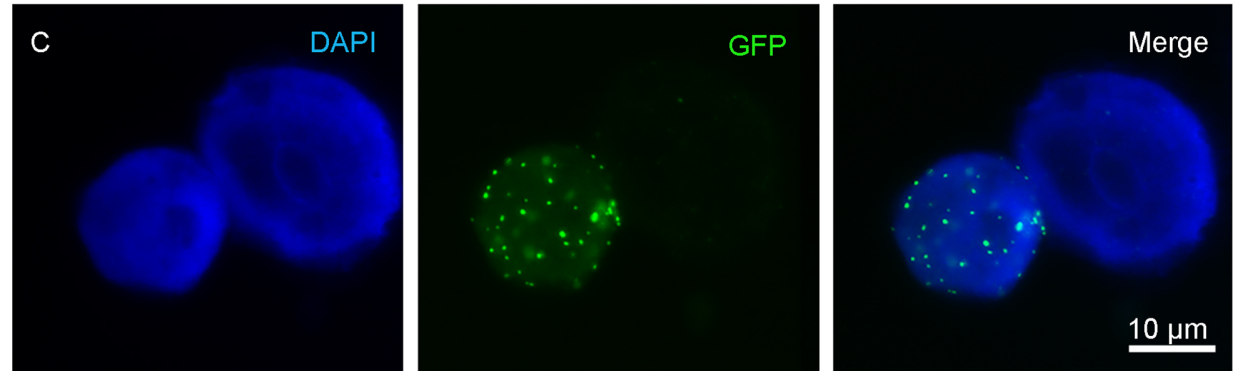

FIGURE 6 | Effect of changing the sgRNA scaffold with a U/A flip and extension on quantity and quality of observed telomere signals. No significant change was observed in the terms of $\mathbf{( A )}$ telomere number or $\mathbf{( B )}$ signal/background noise ratio $(\mathrm{p}<0.05)$. (C) Labeled telomeres by the vector which has the change in sgRNA scaffold. Measurements were performed based on data from 10 isolated nuclei.

\section{DISCUSSION}

\section{Optimization of Aptamer-Based CRISPR Imaging Constructs}

The application of MS2 and PP7 aptamers resulted in improved CRISPR imaging constructs instrumental to trace telomeres in transiently transformed $N$. benthamiana. Labeling efficiency, based on the mean value of signal numbers per nucleus, was increased up to 1.7 fold in comparison to dCas9:GFP. The number of individual telomere signals per nucleus was lower than expected though, which may be due to clustering of individual telomeres. Clustering of telomeres has been also observed in other organisms like A. thaliana (Fransz et al., 2002), yeast and Drosophila melanogaster (Hozé et al., 2013; Wesolowska et al., 2013).

Despite the improved labeling of telomeres, the aptamerbased CRISPR imaging in $N$. benthamiana resulted in a labeling efficiency of $73 \%-75 \%$ compared with FISH. In contrast, in human cell cultures, the number of telomeric signals obtained by CRISPR imaging was almost equal to the number of FISH signals (Chen et al., 2013). The copy number difference of telomere repeats is unlikely the reason for this discrepancy because human telomeres are 5 to $15 \mathrm{~kb}$ (Moyzis et al., 1988) while the telomeres in $N$. benthamiana are 60 to $160 \mathrm{~kb}$ long (Fajkus et al., 1995). Since a temperature of $37^{\circ} \mathrm{C}$ is required for optimal Cas9 activity (Xiang et al., 2017), the temperature difference between plant $\left(22^{\circ} \mathrm{C}\right)$ and mammalian cell cultures $\left(37^{\circ} \mathrm{C}\right)$ might contribute to the observed labeling difference between mammalian and plant species.
While dCas9:GFP expressing cells showed background signals in nucleoli (Dreissig et al., 2017), such background was absent from leaves expressing aptamer-containing reporter constructs. Nucleolar accumulation of dCas9 has been noted in other samples like human cell cultures (Chen et al., 2013). Likely, unspecific labeling of nucleoli was reduced because fluorescent proteins were not directly fused to dCas9.

Substitution of the ubiquitin promoter with the inducible XVE promoter caused a 5-fold decrease in expression of dCas9. However, changing the expression of dCas9 gene by application of XVE promoter did not result in a significant change in the number of observed telomere signals. In contrast, it is demonstrated that the low applied dosage of sgRNA in mammalian cell cultures affects the quality of CRISPR imaging signals (Chen et al., 2013). The PRS5A promoter resulted in a lower number of telomere signals. This could be because PRS5A is more active in meristematic tissues rather than leaves, the tissue which was used for transient transformation (Winter et al., 2007). Regardless of the promoter type, telomeres showed random movement like reported for dCas9:GFP (Dreissig et al., 2017).

Increasing the number of MS2 aptamers to 16 copies did not enhance the efficiency of telomere labeling in N. benthamiana, although in human cell cultures increment of aptamer numbers up to 16 improved labeling (Qin et al., 2017). Additionally, changing the sgRNA scaffold did not increase the quantity and quality of observed signals. In human cell cultures though, similar modifications increased the number of CRISPR-labeled telomeres and improved the signal/background noise (Chen et al., 2013). Fujimoto and Matsunaga (2017) used sgRNA 

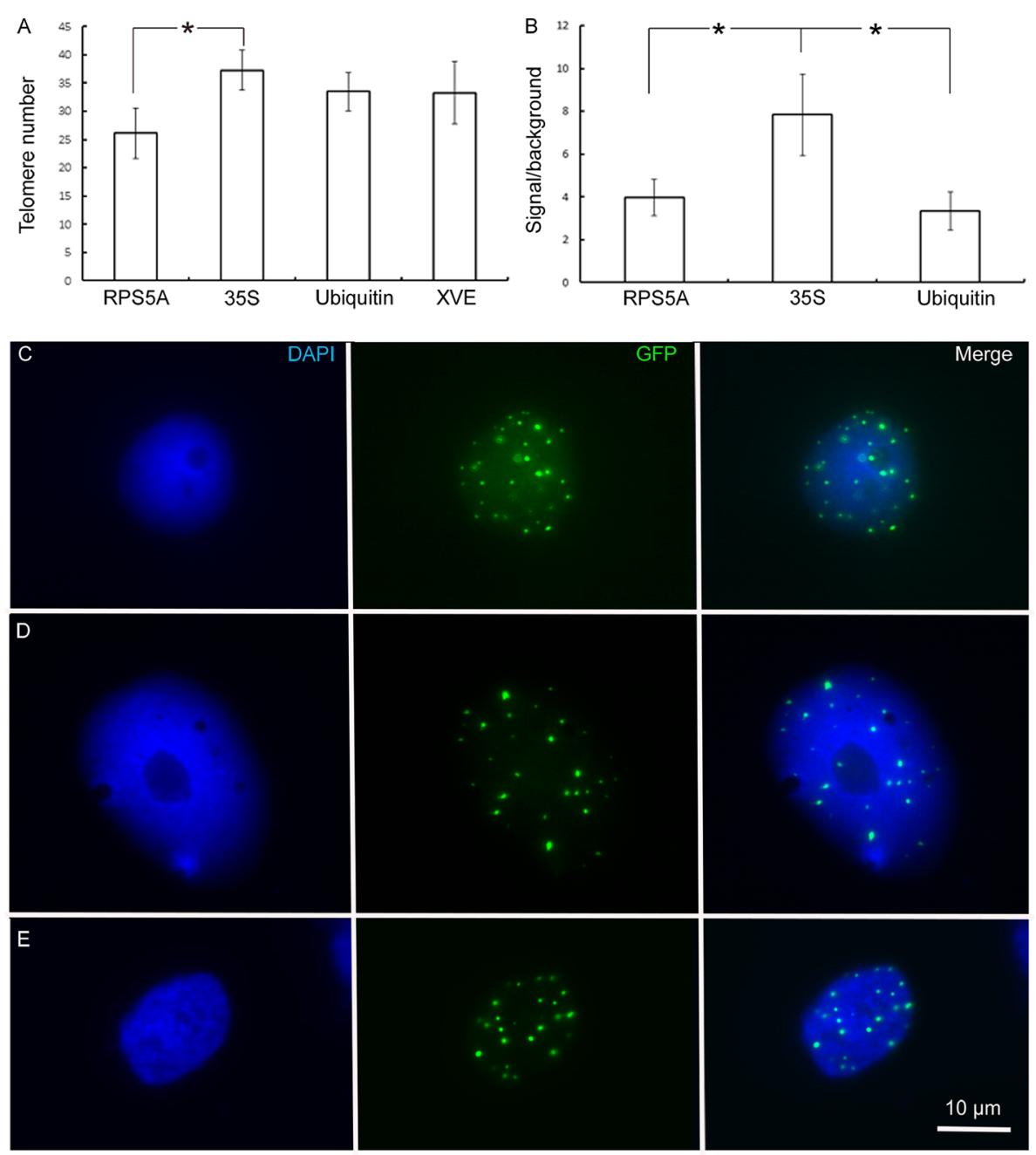

FIGURE 7 | Effect of different promotors used for expression of dCas9 on the efficiency of telomere labeling. (A) The expression of dCas9 by PRS5A promoter resulted in the recognition of a smaller number of telomeres compared to $35 \mathrm{~S}$ and ubiquitin promoters. The XVE inducible promoter was as efficient as ubiquitin promoter regarding the number of labeled telomeres $(p<0.05)$. (B) 35 S promoter caused the better signal to background noise ratio $(p<0.05)$. Data obtained from 10 isolated nuclei per construct. Regardless of promoter type, dCas9 driven by (C) RPS5A, (D) 35S, (E) XVE could label telomeric regions in N. benthamiana.

scaffold modifications ( $\mathrm{T}$ to $\mathrm{G}$ change and $\mathrm{A} / \mathrm{U}$ flip combined with UGCUG extension) within a CRISPR imaging construct to improve the signal to noise ratio of telomere labeling in transiently transformed N. tabacum. The different outcome reported here might be due to the different constructs used.

\section{Why Does CRISPR Imaging Not Work in Stably Transformed Plants?}

Our CRISPR imaging constructs which were successfully applied in transiently transformed $N$. benthamiana leaves could not be used to label defined sequences in stably transformed $N$. benthamiana, A. thaliana or D. carota. The same observation was made by (Fujimoto and Matsunaga, 2017) for GFP-fused dCas9 imaging constructs. Intriguingly, CRISPR-imaging of centromeric and telomeric repeats works-fine on fixed nuclei and chromosomes of different plant and animal species (Deng et al., 2015; Ishii et al., 2019; Nemeckova et al., 2019; Potlapalli et al., 2020). The in situ imaging method CRISPR-FISH (also called REGEN-ISL) is based on a fluorescence-labeled two-part guide RNA with a recombinant Cas9 endonuclease complex. For both imaging methods, we used telomere- and centromerespecific gRNA and A. thaliana and N. benthamiana, subsequently (Ishii et al., 2019), this work). Hence, our expectation was that the selected gRNA in combination with dCas9 should also work in stably transformed plants.

Why then did CRISPR imaging fail in stably transformed plants? In contrast to CRISPR-based editing, for CRISPR imaging a constant interaction of the RNP complex with the target DNA is a functional prerequisite. It is tempting to speculate that a permanent binding of the RNP complex with its target DNA interferes with processes required for plant development. The formation of R-loops, which is underlying 
A

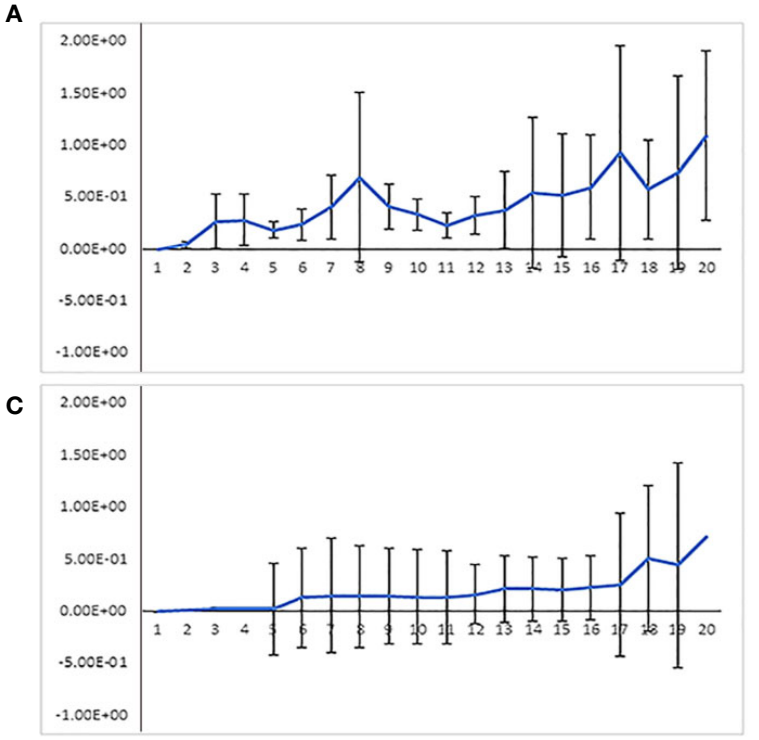

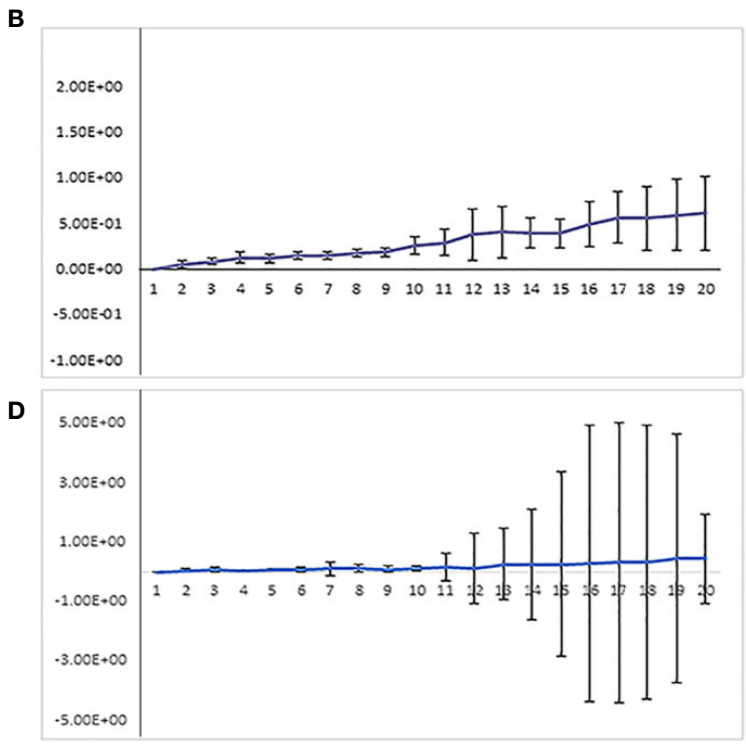

FIGURE 8 | Comparing mean square distance (MSD in $\mu \mathrm{m}$ ) of telomeres labeled by indirectly labeled aptamer-dCas9 which were under the control of (A) $35 \mathrm{~S}$, (B) RPS5a, or (C) ubiquitin promoters. (D) Directly labeled dCas9, which was under the control of a ubiquitin promoter. Telomeres showed random movements regardless of promoter type and how dCas9 was labeled.

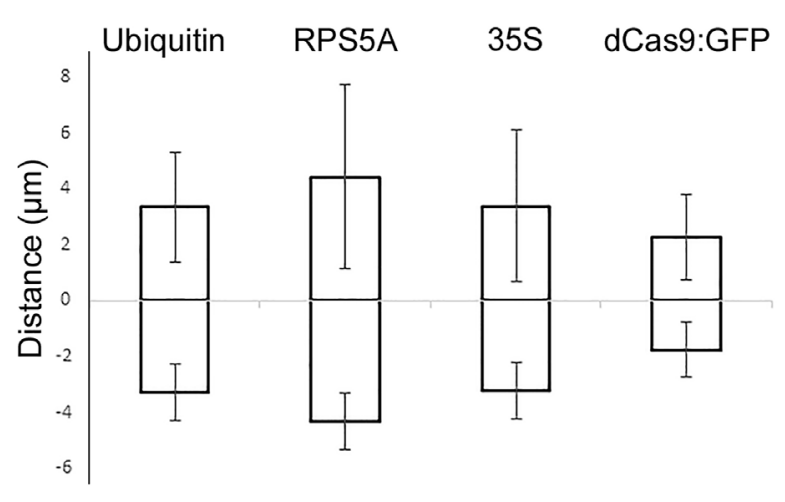

FIGURE 9 | Measurement of inter-telomeric distance changes in nuclei transformed with three different indirectly labeled aptamer-dCas9 which were under the control of ubiquitin, RPS5a or 35S promoters and directly labeled dCas9 which was under the control of the ubiquitin promoter. Intra-telomeric distance changes vary between minimum $\pm 1 \mu \mathrm{m}$ to maximum $\pm 4 \mu \mathrm{m}$.

the CRISPR/Cas mechanism, might hamper cellular processes. R-loops are three-stranded nucleic acid structures composed of a DNA-RNA hybrid and a displaced single-stranded DNA. Rloops have a role in transcription, chromatin modification, DNA damage response. Once the R-loop homeostasis is perturbed, it can lead to genome instability (Crossley et al., 2019; Xu et al., 2020). The R-loop distribution atlas of $A$. thaliana has shown that R-loop distribution patterns are relatively preserved during different developmental and environmental conditions ( $\mathrm{Xu}$ et al., 2020). Therefore, by imposing consistent formation of R-loops in targeted regions, CRISPR imaging constructs might change Rloop dynamics in defined genomic regions of stably transformed plants. Alternatively, the selected Cas9 variant of S. pyogenes is not suitable and further optimized Cas variants with higher efficiency could overcome this problem. A negative selection against CRISPR-imaging constructs in stably transformed plants at the transcript level is less likely because corresponding transcripts exist. In addition, uniform labeling of anti-Cas9 immunosignals was detected in transformed plants. Overcoming the discussed problem will also help to increase the efficiency of CRISPR-based editing in plants.

Taking advantage of the intrinsic stability of CRISPR guide RNA, (Wang et al., 2019) used fluorescent ribonucleoproteins consisting of chemically synthesized fluorescent gRNAs and recombinant dCas9 protein for imaging in transfected living human lymphocytes. Live-cell fluorescent in situ hybridization (LiveFISH) allowed tracking of multiple chromosomal loci in lymphocytes. Whether the transient transformation of cells with fluorescent RNP complexes could become another option to label defined sequences in living plant cells remains to be demonstrated.

\section{CONCLUSIONS}

A three-component labeling method using dCas9, PP7/MS2 aptamers and tdMCP : GFP/tdPCP : GFP binding to MS2/PP7 aptamers was successfully applied for labeling of telomeres in transiently transformed $N$. benthamiana. The labeling efficiency of telomeres was increased and the background labeling noise in the nucleolus was reduced compared to previous work (Dreissig 
et al., 2017). The copy number of aptamers used in the aptamerbased imaging construct is critical. The level of $d \operatorname{Cas} 9$ gene expression does not affect CRISPR imaging. The application of CRISPR/Cas9 for live-cell imaging in stably transformed plants, however, was not successful.

\section{DATA AVAILABILITY STATEMENT}

All datasets presented in this study are included in the article/ Supplementary Material.

\section{AUTHOR CONTRIBUTIONS}

SK contributed in conducting lab work needed for stated experiments in the manuscript. PS contributed in conducting lab work needed for preparing required imaging vectors. EG participated in analysis of telomere signals. FD performed the Daucus carota transformation with live-imaging vectors. TR performed confocal microscopy. $\mathrm{HP}$ and $\mathrm{AH}$ initiated and supervised the project. All authors wrote the manuscript.

\section{FUNDING}

The work was funded by Deutsche Forschungsgemeinschaft (DFG) grant HO1779/28-1.

\section{ACKNOWLEDGMENTS}

We would like to thank Sabine Struckmeyer, Christine Helmold, Oda Weiß, Christin-Sophie Gäde, and Sylvia Swetik for technical support, Steven Dreissig for scientific discussion and Christian Hertig for preparing schemata for the manuscript and the statistical analysis with SigmaStat 4.0. We also thank Michael $\mathrm{M}$. Goodin for providing N. benthamiana seeds expressing CFPH2B, Bruno Müller for a plasmid containing the RPS5A promoter, Chua Nam Hai for the pER8 containing XVE promoter, Martina Dvořácková and Jiri Fajkus for plasmid expressing TRB1-GFP. The work was funded by Deutsche Forschungsgemeinschaft (DFG) grant HO1779/28-1.

\section{REFERENCES}

Chen, B., Gilbert, L. A., Cimini, B. A., Schnitzbauer, J., Zhang, W., Li, G. W., et al. (2013). Dynamic imaging of genomic loci in living human cells by an optimized CRISPR/Cas system. Cell 155, 1479-1491. doi: 10.1016/j.cell. 2013.12.001

Clemente, T. (2006). "Nicotiana (Nicotiana tobaccum, Nicotiana benthamiana)," in Agrobacterium Protocols. Ed. K. Wang (Totowa, NJ: Hummana Press), 153-154.

Crossley, M. P., Bocek, M., and Cimprich, K. A. (2019). R-Loops as cellular regulators and genomic threats. Mol. Cell 73, 398-411. doi: 10.1016/j.molcel. 2019.01.024

Deng, W. L., Shi, X. H., Tjian, R., Lionnet, T., and Singer, R. H. (2015). CASFISH: CRISPR/Cas9-mediated in situ labeling of genomic loci in fixed cells. Proc. Natl. Acad. Sci. U. S. A. 112, 11870-11875. doi: 10.1073/pnas.1515692112
This manuscript has been released as a pre-print at BIORXIV (MS ID\#: BIORXIV/2020/078246).

\section{SUPPLEMENTARY MATERIAL}

The Supplementary Material for this article can be found online at: https://www.frontiersin.org/articles/10.3389/fpls.2020.01254/ full\#supplementary-material

MOVIE SUPPLEMENT 1 | Co-expression of dCas9:2xMS2:mRuby (red) and TRB1 (green) in N. benthamina. Co-localization of telomeric dCas9:2xMS2:mRuby and TRB1 shows that aptamer-based imaging construct can be also used for DNAprotein interaction studies.

MOVIE SUPPLEMENT 2 | Dynamic of telomeres targeted by indirectly labeled dCas9 with MS2 aptamer in N. benthamiana leaf nuclei expressing CFP-H2B.

FIGURE SUPPLEMENT 1 | Different components of the aptamer-based labeling method: 1) dCas9 from S. pyogenes, 2) MS2 or PP7 aptamers (here only MS2 is shown) which are integrated into sgRNA scaffold. 3) RNA binding protein (tdMCP or tdPCP) fused to a fluorescent protein (mRuby) which recognizes aptamers.

FIGURE SUPPLEMENT 2 | Changing the sgRNA scaffold with A U flip (in red) and insertion of an extension (in green).

FIGURE SUPPLEMENT 3 | Specificity control test by ImmunoFISH for the activity of the inducible XVE promoter. (A) Isolated nuclei after treatment of leaves with $\beta$-estradiol show telomeric signals. Co-localization of dCas9:2xMS2:GFP and FISH signals show that the observed signals are telomeric specific. (B) Nuclei isolated from $\beta$-estradiol-untreated leaves show a uniform labeling of nuclei.

FIGURE SUPPLEMENT 4 | Real time expression of dCas9 expressed by ubiquitin and XVE promoters. dCas9 expression is much lower when it is driven by inducible XVE promoter compared to ubiquitin from parsley. Error bars are standard deviation.

FIGURE SUPPLEMENT 5 | Selected nuclei of $A$. thaliana stably transformed with a centromere-specific dCas9:2xMS2:GFP construct exhibiting dot-like signals. (A, B) Application of the centromere-specific protospacer 1 and 2, respectively. The number of signals was higher than expected.

FIGURE SUPPLEMENT 6 | Immunostaining of dCas9 protein in isolated nucle from leaf material of stably transformed Arabidopsis plants with dCas9:2xMS2: GFP targeting centromeric regions. (A) Immunostaining of dCas9 in stably transformed Arabidopsis plants showed the dCas9 is not degraded. (B) Immunostaining of isolated leaf nuclei from wild type Arabidopsis leaf nuclei did not result in signals which shows that the applied antibody against dCas 9 is working specifically.

Doležel, J., Greilhuber, J., and Suda, J. (2007). Estimation of nuclear DNA content in plants using flow cytometry. Nat. Protoc. 2, 2233-2244. doi: 10.1038/ nprot. 2007.310

Dreissig, S., Schiml, S., Schindele, P., Weiss, O., Rutten, T., Schubert, V., et al. (2017). Live-cell CRISPR imaging in plants reveals dynamic telomere movements. Plant J. 91, 565-573. doi: 10.1111/tpj.13601

Dunemann, F., Unkel, K., and Sprink, T. (2019). "Using CRISPR/Cas9 to produce haploid inducers of carrot through targeted mutations of centromeric histone H3 (CENH3)," in II International Symposium on Carrot and Other Apiaceae Eds. D. Grzebelus and R. Barański (ISHS Acta Horticulturae), 211-219.

Esvelt, K. M., Mali, P., Braff, J. L., Moosburner, M., Yaung, S. J., and Church, G. M. (2013). Orthogonal Cas9 proteins for RNA-guided gene regulation and editing. Nat. Methods 10, 1116. doi: 10.1038/nmeth.2681 
Fajkus, J., Kovařík, A., mKrálovics, R., and Bezděk, M. (1995). Organization of telomeric and subtelomeric chromatin in the higher plant Nicotiana tabacum. Molec. Gen. Genet. 247, 633-638.

Fransz, P., De Jong, J. H., Lysak, M., Castiglione, M. R., and Schubert, I. (2002). Interphase chromosomes in Arabidopsis are organized as well defined chromocenters from which euchromatin loops emanate. Proc. Natl. Acad. Sci. U. S. A. 99, 14584-14589. doi: 10.1073/pnas.212325299

Fu, Y., Rocha, P. P., Luo, V. M., Raviram, R., Deng, Y., Mazzoni, E. O., et al. (2016). CRISPR-dCas9 and sgRNA scaffolds enable dual-colour live imaging of satellite sequences and repeat-enriched individual loci. Nat. Commun. 7, 11707. doi: $10.1038 /$ ncomms 11707

Fujimoto, S., and Matsunaga, S. (2017). Visualization of Chromatin Loci with Transiently Expressed CRISPR/Cas9 in Plants. Cytologia 82, 559-562. doi: 10.1508/cytologia.82.559

Fujimoto, S., Sugano, S. S., Kuwata, K., Osakabe, K., and Matsunaga, S. (2016). Visualization of specific repetitive genomic sequences with fluorescent TALEs in Arabidopsis thaliana. J. Exp. Bot. 67, 6101-6110. doi: 10.1093/jxb/erw371

Hong, Y., Lu, G., Duan, J., Liu, W., and Zhang, Y. (2018). Comparison and optimization of CRISPR/dCas9/gRNA genome-labeling systems for live cell imaging. Genome Biol. 19, 39. doi: 10.1186/s13059-018-1413-5

Horn, B. K. P. (1987). Closed-form solution of absolute orientation using unit quaternions. J. Optical Soc. Am. a-Optics Image Sci. Vision 4, 629-642. doi: 10.1364/JOSAA.4.000629

Hozé, N., Ruault, M., Amoruso, C., Taddei, A., and Holcman, D. (2013). Spatial telomere organization and clustering in yeast Saccharomyces cerevisiae nucleus is generated by a random dynamics of aggregation-dissociation. Mol. Biol. Cell 24, 1791-1800. doi: 10.1091/mbc.e13-01-0031

Ishii, T., Sunamura, N., Matsumoto, A., Eltayeb, A. E., and Tsujimoto, H. (2015). Preferential recruitment of the maternal centromere-specific histone $\mathrm{H} 3$ $(\mathrm{CENH} 3)$ in oat (Avena sativa L.) $\times$ pearl millet (Pennisetum glaucum L.) hybrid embryos. Chromosome Res. 23, 709-718. doi: 10.1007/s10577-0159477-5

Ishii, T., Schubert, V., Khosravi, S., Dreissig, S., Metje-Sprink, J., Sprink, T., et al. (2019). RNA-guided endonuclease - in situ labelling (RGEN-ISL): a fast CRISPR/Cas9-based method to label genomic sequences in various species. New Phytol. 222, 1652-1661. doi: 10.1111/nph.15720

Khosravi, S., Ishii, T., Dreissig, S., and Houben, A. (2020). Application and prospects of CRISPR/Cas9-based methods to trace defined genomic sequences in living and fixed plant cells. Chromosome Res. 28, 7-17. doi: 10.1007/s10577-019-09622-0

Konermann, S., Brigham, M. D., Trevino, A. E., Joung, J., Abudayyeh, O. O., Barcena, C., et al. (2015). Genome-scale transcriptional activation by an engineered CRISPR-Cas9 complex. Nature 517, 583-588. doi: 10.1038/ nature14136

Lee, J. E., Neumann, M., Iglesias Duro, D., and Schmid, M. (2019). CRISPR-based tools for targeted transcriptional and epigenetic regulation in plants. PLoS One 14, e0222778. doi: 10.1371/journal.pone.0222778

Lindhout, B. I., Fransz, P., Tessadori, F., Meckel, T., Hooykaas, P. J. J., and Zaal, B. J. (2007). Live cell imaging of repetitive DNA sequences via GFP-tagged polydactyl zinc finger proteins. Nucleic Acids Res. 35, e107. doi: 10.1093/nar/ gkm618

Ma, H., Naserib, A., Reyes-Gutierreza, P., Wolfec, S. A., Zhangb, S., and Pedersona, T. (2015). Multicolor CRISPR labeling of chromosomal loci in human cells. PNAS 112, 3002-3007. doi: 10.1073/pnas.1420024112

Ma, H., Tu, L. C., Naseri, A., Huisman, M., Zhang, S., Grunwald, D., et al. (2016). Multiplexed labeling of genomic loci with dCas 9 and engineered sgRNAs using CRISPRainbow. Nat. Biotechnol. 34, 528-530. doi: 10.1038/nbt.3526

Martin, K., Kopperud, K., Chakrabarty, R., Banerjee, R., Brooks, R., and Goodin, M. M. (2009). Transient expression in Nicotiana benthamiana fluorescent marker lines provides enhanced definition of protein localization, movement and interactions in planta. Plant J. 59, 150-162. doi: 10.1111/j.1365313X.2009.03850.x

Misteli, T. (2007). Beyond the Sequence: Cellular Organization of Genome Function. Cell 128, 787-800. doi: 10.1016/j.cell.2007.01.028

Moyzis, R. K., Buckingham, J. M., Cram, L. S., Dani, M., Deaven, L. L., Jones, M. D., et al. (1988). A highly conserved repetitive DNA sequence, (TTAGGG) n, present at the telomeres of human chromosomes. Proc. Natl. Acad. Sci. U. S. A. 85, 6622-6626. doi: 10.1073/pnas.85.18.6622
Nemeckova, A., Wasch, C., Schubert, V., Ishii, T., Hribova, E., and Houben, A. (2019). CRISPR/Cas9-based RGEN-ISL allows the simultaneous and specific visualization of proteins, DNA repeats, and sites of DNA replication. Cytogenet. Genome Res. 159, 48-53. doi: 10.1159/000502600

Phan, H. T., and Conrad, U. (2016). "Plant-Based Vaccine Antigen Production," in Vaccine Technologies for Veterinary Viral Diseases: Methods and Protocols. Ed. A. Brun (New York, NY: Springer New York), 35-47.

Potlapalli, B. P., Schubert, V., Metje-Sprink, J., Liehr, T., and Houben, A. (2020). Application of Tris- $\mathrm{HCl}$ allows the specific labeling of regularly prepared chromosomes by CRISPR-FISH. Cytogenet. Genome Res. 160, 156-165. doi: $10.1159 / 000506720$

Qin, P., Parlak, M., Kuscu, C., Bandaria, J., Mir, M., Szlachta, K., et al. (2017). Live cell imaging of low- and non-repetitive chromosome loci using CRISPR-Cas9. Nat. Commun. 8, 14725. doi: 10.1038/ncomms 14725

Robinett, C. C., Straight, A. F., Li, G. W., Willhelm, C., Sudlow, G., Murray, A., et al. (1996). In vivo localization of DNA sequences and visualization of largescale chromatin organization using lac operator/repressor recognition. J. Cell Biol. 135, 1685-1700. doi: 10.1083/jcb.135.6.1685

Saad, H., Gallardo, F., Dalvai, M., Tanguy-Le-Gac, N., Lane, D., and Bystricky, K. (2014). DNA dynamics during early double-strand break processing revealed by non-intrusive imaging of living cells. PLoS Genet. 10 (3), e1004187. doi: 10.1371/journal.pgen.1004187

Schrumpfová, P. P., Vychodilová, I., Dvořáčková, M., Majerská, J., Dokládal, L., Schořová, S., et al. (2014). Telomere repeat binding proteins are functional components of Arabidopsis telomeres and interact with telomerase. Plant J. 77, 770-781. doi: 10.1111/tpj.12428

Selma, S., Bernabé-Orts, J. M., Vazquez-Vilar, M., Diego-Martin, B., Ajenjo, M., Garcia-Carpintero, V., et al. (2019). Strong gene activation in plants with genome-wide specificity using a new orthogonal CRISPR/Cas9-based programmable transcriptional activator. Plant Biotechnol. J. 17, 1703-1705. doi: $10.1111 /$ pbi.13138

Shao, S., Zhang, W., Hu, H., Xue, B., Qin, J., Sun, C., et al. (2016). Long-term dualcolor tracking of genomic loci by modified sgRNAs of the CRISPR/Cas9 system. Nucleic Acids Res. 44, e86. doi: 10.1093/nar/gkw066

Shechner, D., Hacisuleyman, E., Younger, S., and Rinn, J. L. (2015). Multiplexable, locus-specific targeting of long RNAs with CRISPR-Display. Nat. Methods 12, 664670. doi: 10.1038/nmeth.3433

Tanenbaum, M. E., Gilbert, L. A., Qi, L. S., Weissman, J. S., and Vale, R. D. (2014). A protein tagging system for signal amplification in gene expression and fluorescence imaging. Cell 159, 635. doi: 10.1016/j.cell.2014.09.039

Tepfer, M., Gaubert, S., Leroux-Coyau, M., Prince, S., and Houdebine, L. (2004). Transient expression in mammalian cells of transgenes transcribed from the Cauliflower mosaic virus $35 \mathrm{~S}$ promoter. Environ. Biosaf. Res. 3, 91-97. doi: 10.1051/ebr:2004010

Urbanek, M. O., Galka-Marciniak, P., Olejniczak, M., and Krzyzosiak, W. J. (2014). RNA imaging in living cells - methods and applications. RNA Biol. 11, 1083-1095. doi: 10.4161/rna.35506

Wang, S., Su, J. H., Zhang, F., and Zhuang, X. (2016). An RNA-aptamer-based two-color CRISPR labeling system. Sci. Rep. 6, 26857. doi: 10.1038/ srep 26857

Wang, H., Nakamura, M., Zhao, D., Nguyen, C. M., Yu, C., Lo, A., et al. (2019). Temporal-Spatial Visualization of Endogenous Chromosome Rearrangements in Living Cells. Science 365 (6459), 1301-1305. doi: 10.1101/734483

Weijers, D., Franke-Van Dijk, M., Vencken, R. J., Quint, A., Hooykaas, P., and Offringa, R. (2001). An Arabidopsis Minute-like phenotype caused by a semidominant mutation in a RIBOSOMAL PROTEIN S5 gene. Development 128, 42894299.

Wesolowska, N., Amariei, F. L., and Rong, Y. S. (2013). Clustering and Protein Dynamics of Drosophila melanogaster Telomeres. Genetics 195, 381-391. doi: 10.1534/genetics.113.155408

Winter, D., Vinegar, B., Nahal, H., Ammar, R., Wilson, G. V., and Provart, N. J. (2007). An "Electronic Fluorescent Pictograph" browser for exploring and analyzing large-scale biological data sets. PLoS One 2, e718. doi: 10.1371/ journal.pone.0000718

Wu, X., Mao, S., Ying, Y., Krueger, C. J., and Chen, A. K. (2019). Progress and Challenges for Live-cell Imaging of Genomic Loci Using CRISPR-based Platforms. Genomics Proteomics Bioinf. 17, 119-128. doi: 10.1016/j.gpb. 2018.10.001 
Xiang, G., Zhang, X., An, C., Cheng, C., and Wang, H. (2017). Temperature effect on CRISPR-Cas9 mediated genome editing. J. Genet. Genomics 44, 199-205. doi: 10.1016/j.jgg.2017.03.004

Xu, W., Li, K., Li, S., Hou, Q., Zhang, Y., Liu, K., et al. (2020). The R-loop Atlas of Arabidopsis Development and Responses to Environmental Stimuli. Plant Cell. 32, 888-903. doi: $10.1105 /$ tpc. 19.00802

Zhang, S., and Song, Z. (2017). Aio-Casilio: a robust CRISPR-Cas9-Pumilio system for chromosome labeling. J. Mol. Histol. 48, 293-299. doi: 10.1007/s10735-017-9727-2

Zuo, J., Niu, Q.-W., and Chua, N.-H. (2000). An estrogen receptor-based transactivator XVE mediates highly inducible gene expression in transgenic plants. Plant J. 24, 265-273. doi: 10.1046/j.1365-313x.2000.00868.x
Conflict of Interest: The authors declare that the research was conducted in the absence of any commercial or financial relationships that could be construed as a potential conflict of interest.

Copyright $\odot 2020$ Khosravi, Schindele, Gladilin, Dunemann, Rutten, Puchta and Houben. This is an open-access article distributed under the terms of the Creative Commons Attribution License (CC BY). The use, distribution or reproduction in other forums is permitted, provided the original author(s) and the copyright owner(s) are credited and that the original publication in this journal is cited, in accordance with accepted academic practice. No use, distribution or reproduction is permitted which does not comply with these terms. 